\title{
Albumin/Globulin Ratio as Yin-Yang in Rheumatoid Arthritis and Its Correlation to Inflamm-Aging Cytokines
}

\author{
Yong Chen (1) \\ Yanjuan Chen ${ }^{2}$ \\ Li Zhao ${ }^{3}$ \\ $\mathrm{Hui} \mathrm{He}{ }^{3}$ \\ Laiyou Wei ${ }^{1}$ \\ Wenjuan Lai' \\ Jingxia Yuan' \\ Xiaoping Hong' \\ Lixiong Liu' \\ Baojiang Wang ${ }^{4}$ \\ Kutty Selva Nandakumar ${ }^{5, *}$ \\ Dongzhou Liu',*
}

'Division of Rheumatology and Research, The Second Clinical Medical College, Jinan University (Shenzhen People's

Hospital), Shenzhen, Guangdong, People's

Republic of China; ${ }^{2}$ School of Basic

Medicine, Jinan University, Guangzhou,

Guangdong, People's Republic of China;

${ }^{3}$ Division of Health Management, The

Second Clinical Medical College, Jinan

University (Shenzhen People's Hospital),

Shenzhen, Guangdong, People's Republic

of China; ${ }^{4}$ Shenzhen Maternity \& Child

Healthcare Hospital, Shenzhen,

Guangdong, People's Republic of China;

${ }^{5}$ Southern Medical University-Karolinska Institute United Medical Inflammation Center, Southern Medical University,

Guangzhou, Guangdong, People's

Republic of China

*These authors contributed equally to this work

Correspondence: Dongzhou Liu

Division of Rheumatology and Research,

The Second Clinical Medical College, Jinan

University (Shenzhen People's Hospital),

Shenzhen, Guangdong, People's Republic

of China

Tel +86 I83 2III 6879

Email liu_dz2001@sina.com
Purpose: Inflamm-aging is a novel-concept in rheumatoid arthritis (RA) with accelerating aging process. We try to find a correlation between serum albumin/globulin (A/G) ratio and clinical biochemical parameters, incidence of aging-related diseases (ARDs) as well as inflammaging-related molecules.

Patients and Methods: Healthy controls (HC) and RA patients were compared with their clinical biochemical parameters including albumin and globulin levels, $\mathrm{A} / \mathrm{G}$ ratio, and levels of serum lipids. Incidence of ARDs in RA was compared with $\mathrm{A} / \mathrm{G}$ ratio, having a cut off value of 1.2. Expression levels of leptin and Trf2 genes in PBMCs, and inflammatory factors like IL-1 $\beta$, IL-6, IL-8 and TNF-a between HC and RA patients were compared, and correlated with the $\mathrm{A} / \mathrm{G}$ ratio.

Results: Compared to HC, RA patients had decreased levels of albumin, while globulin levels were found to be increased, which led to a significantly lower $\mathrm{A} / \mathrm{G}$ ratio in RA patients. $\mathrm{A} / \mathrm{G}$ ratio rather than ESR and $\mathrm{CRP}$ had significant correlation with dyslipidemia in RA patients. Patients with $\mathrm{A} / \mathrm{G}<1.2$ had a higher risk of ARDs than patients with $\mathrm{A} / \mathrm{G}$ $>1.2$. The RR was 2.48 (95\% CI: 1.79 to 3.64, $\mathrm{p}<0.0001)$. In addition, $\mathrm{A} / \mathrm{G}$ ratio has positively correlated to leptin and Trf2 expression, while an inverse correlation was observed with the levels of inflamm-aging related cytokines like IL-6, IL-8 and TNF-a.

Conclusion: A decreased A/G ratio in RA patients has significantly correlated with dyslipidemia and ARDs, as well as inflammaging- related adipokine and pro-inflammatory cytokines. Thus, A/G ratio could be a reliable marker for evaluating the inflammaging process during clinical management in ARDs.

Keywords: inflammation, age-related disease, dyslipidemia, leptin, biomarker

\section{Introduction}

Rheumatoid arthritis (RA) is one of the most common, systemic, chronic autoimmune diseases (AID). Besides bone and cartilage of joints being most susceptible tissues, other organs could also be involved in the disease manifestations, including anaemia, osteoporosis, infection, and damage to skin or lungs. ${ }^{1}$ The concept of inflamm-aging has gained ground because chronic inflammation proved to be one of the mechanisms that could contribute to the pathogenesis of senility and age-related diseases (ARDs). ${ }^{2}$ Evidence from clinical observations suggested that patients with AID like RA, systemic lupus erythematosus, ankylosing spondylitis and Behcet's disease, exhibit susceptibility to ARDs, especially cardiovascular disease (CVD), diabetes etc. ${ }^{3,4}$ 
Leptin regulates both innate and adaptive immunity mainly through pro-inflammatory effects. In autoimmune diseases, leptin resistance is observed, which has a role in the generation and maintenance of autoimmunity. ${ }^{5} \operatorname{Trf} 2$ is a telomere associated protein having a crucial role in the maintenance of telomere. ${ }^{6}$ Pro-inflammatory cells and cytokines that participate in the inflamm-aging process have been broadly investigated. ${ }^{7}$ Nevertheless, a recognized clinical marker is currently lacking that could be applied in daily clinical practice to interpret the inflamm-aging process. In daily clinical work, the inner cause and effect of RA and ARDs reflecting the inflamm-aging process are usually neglected. Therefore, an accessible clinical index will help in identifying ARDs concomitant to AIDs that can lead to prevention of progress in inflamm-aging.

Inflammation increases permeability of capillaries causing escape of serum albumin into interstitial space. Although fractional synthesis of albumin increases due to decreased plasma level, the half-life of albumin tends to shorten significantly, which further decreases albumin levels. Therefore, hypoalbuminemia results from and reflects the inflammatory state. It is associated with poor quality of life and reduced longevity. ${ }^{8}$ Conversely, high globulin level indicates inflammatory conditions related to viral and bacterial infections, as well as inflammatory or immune disorders. ${ }^{9}$ From the perspective of physiology and pathology, one can assign the pair of albumin and globulin levels to Yin and Yang, which indicate dual sides of the immune balance. Albumin/globulin (A/G) ratio is a classic clinical index that can provide unspecific information about various diseases. For example, recent studies explored its correlation with the survival and mortality of various cancers, uremia, and heart failure, ${ }^{10-12}$ as well as with ARDs like chronic obstructive pulmonary disease, cardiovascular diseases, and cognitive disorders..$^{13,14}$

It is documented that most if not all ARDs and even other internal diseases share inflammatory pathways. ${ }^{15,16}$ Interestingly, traditional Chinese medicine (TCM) theory put forward by Zhu Danxi (1281-1358) in ancient China described the pathogenesis of common internal diseases as Yin is insufficient, while Yang is in surplus. ${ }^{17}$ Based on this knowledge, we hypothesize that $\mathrm{A} / \mathrm{G}$ might also be involved in the prognosis of RA. Thus, by correlating the $\mathrm{A} / \mathrm{G}$ ratio with ARDs and inflammatory cytokines, we can explore the Yin-Yang nature of $\mathrm{A} / \mathrm{G}$, and also its potential linkage with the inflamm-aging process.

\section{Materials and Methods}

\section{Study Design and Participants}

ust 2019 to March 2021 with RA patients $(\mathrm{n}=134)$ recruited from Rheumatology Department and healthy controls $(\mathrm{HC}, \mathrm{n}=38)$ from Health Management Center of Shenzhen People's Hospital, The Second Clinical Medical College of Jinan University, China. As gender plays an important role in aging, ${ }^{18}$ and the incidence of RA is around $80 \%$ in females, with multiple manifestations, including disease activity and co-morbidities that are different from male patients, ${ }^{19}$ we enrolled only females in this study. The other inclusion and exclusion criteria for RA patients were as follows: (1) patients should strictly meet the 2010 ACR/EULAR classification criteria for rheumatoid arthritis, ${ }^{20}$ and those with overlapping AIDs such as SLE and Sjögren syndrome were excluded; (2) patients having full medical records with present and past history and biochemical test results were included. This information were accessed from hospital information system (HIS); (3) patients voluntarily donating plasma samples and peripheral blood mononuclear cells (PBMCs) for storage at $-80^{\circ} \mathrm{C}$ in the biological sample bank were included; (4) patients with infection, liver and kidney diseases, onset of allergy reactions or medical conditions or blood transfusion that would affect albumin and globulin levels were excluded; (5) patients who had had strokes, cardiovascular events, cancer-associated cachexia or severe acid-based balance disorders and needed intensive care were not included. All participants were at least 18 years of age and gave informed consent for this study, which was approved by the Ethics Committee of Shenzhen People's Hospital, The Second Clinical Medical College of Jinan University, China in accordance with the Declaration of Helsinki.

Medical records of RA patients were reviewed, their age and clinical laboratory findings including plasma levels of albumin, globulin, A/G ratio, total cholesterol (TC), triglycerides (TG), low-density lipoprotein (LDL), high-density lipoprotein (HDL) and uric acid levels were compared with HC. Correlations between the levels of albumin, globulin and $A / G$ ratio with serum lipids in RA patients were analyzed. Differences between RA with or without cardiovascular disease, including high blood pressure (above grade 2), coronary heart disease, heart failure, stroke and atherosclerosis were compared. Incidence of ARDs including CVD, type 2 diabetes mellitus (DM), cancer and osteoporosis (OP) having A/G less or above 
1.2 were compared. The mRNA expression of leptin and Trf2 in PBMCs was compared between RA and HC, and their correlation with $\mathrm{A} / \mathrm{G}$ ratio in RA was analyzed. Nerve growth factor (NGF), macrophage chemoattractant protein-1 (MCP-1), IL-1 $\beta$, IL-6, IL-8 and TNF-a levels were examined by an endocrine multiplex assay using 46 randomly selected RA patients and all $\mathrm{HC}$ individuals. The flowchart of the study is given in Supplementary Figure 1.

\section{Clinical Information and Biochemical Tests}

Current and past medical history of RA patients was reviewed and collected from HIS data including information on age and comorbidities. Blood samples were taken at the end of a $12 \mathrm{~h}$ fasting period after admission. Blood samples from $\mathrm{HC}$ were taken similarly in the Health Management Center. ESR was measured by Westergren method; CRP, albumin, globulin, A/G, TC, TG, LDL, HDL and UA were measured using Roche cobas 702 automatic biochemical analyzer. The serum globulin concentration was calculated by subtracting albumin concentration from total protein amount. The normal range of $\mathrm{A} /$ $\mathrm{G}$ ratio was found to be between 1.2 and 2.0.

\section{RNA Isolation and $\mathrm{qPCR}$}

Five milliliters of peripheral blood from each participant was collected in a vacuum collection tube containing EDTA-K2 (Kangjie, China). PBMCs were obtained by density gradient sedimentation method using human lymphocyte separation medium (Dakewe, China). Total RNA was isolated using TRIzol ${ }^{\circledR}$ Reagent (Thermo Scientific, USA) and quantified using Qubit (Thermo Fisher, USA). RNA was reversetranscribed into cDNA using PrimeScript ${ }^{\mathrm{TM}}$ RT Master Mix (Takara, Japan). The reaction mixture contained $5 \mu \mathrm{L}$ of 2 x TB Green Premix Ex Taq II (Takara, Japan), $3 \mu \mathrm{L}$ of nuclease-free water, $1 \mu \mathrm{L}$ of cDNA, $0.4 \mu \mathrm{L}$ of each genespecific primer and $0.2 \mu \mathrm{L}$ of ROX Reference Dye. The qRTPCR analysis was performed using Applied Biosystems ViiA $^{\text {TM }} 7$ Real-Time PCR System (Thermo Fisher, USA). Each value represents an average from two independent biological replicates. The GAPDH gene expression was used for data standardization. The fold change was calculated using delta $\mathrm{CT}(\Delta \mathrm{Ct})$ method. Primers of GAPDH, leptin and Trf2 are given in Supplementary Table 1.

\section{Luminex Assay}

The peripheral blood samples obtained were centrifuged at $2000 \mathrm{rpm}$ for $8 \mathrm{~min}$ to collect the plasma samples. Each plasma sample was analyzed for NGF, MCP-1, IL-1, IL-6,
IL-8 and TNF-a levels by Luminex bead-based MILLIPLEX $^{\circledR}$ assay using human adipokine magnetic bead panel kit (Millipore, USA) with MILLIPLEX ${ }^{\circledR}$ MAGPIX platform. Data were analyzed using the MILLIPLEX Analyst.V5.1 software.

\section{Statistical Analysis}

Based on our previous investigation, compared to DAS28ESR, A/G ratio indicated disease activity in RA with a false negative rate of $40 \%(17 / 42),{ }^{21}$ so the diagnostic power would be considered as $0.6(1-0.4)$. By setting power $=0.6$, $a=0.05, \rho 0=0.0$, and $\rho 1=0.4$ in the Pearson's correlation test of PASS 15.0.5 software (USA), the minimum size of the sample that needed to be used was calculated as 30 . Since we proposed to evaluate the feasibility of using $A / G$ ratio to significantly correlate with the disease activity, ARDs comorbidities and production of inflammatory cytokines, we recruited as many cases as possible for this pilot study. The "cor" function in the $\mathrm{R}$ language was used to analyze the Pearson correlation coefficient between each variable from RA patients. The corrplot package in $\mathrm{R}$ was used to visualize the heat map of the correlation coefficient values (Supplementary Figure 2). Interesting correlations like the levels of albumin, globulin, $\mathrm{A} / \mathrm{G}$ ratio and other indexes were further analyzed with linear regression and other correlation tests using Prism software. The measurement data were expressed with " $\mathrm{r}$ " at $95 \%$ confidence interval (CI). Differences in age, laboratory tests, relative mRNA expression levels and cytokines between RA and HC, and also between RA with or without CVD, were compared using variance analysis or $t$-test for data with normal distribution. If normal distribution was not observed, then a nonparametric method was used for the analysis. Data were expressed as mean \pm standard deviation (SD). The incidence rate of RA with or without $\mathrm{ARDs}$, at $\mathrm{A} / \mathrm{G}$ ratio less than 1.2 and above 1.2, were analyzed by chi-square (or Fisher's exact) test based on the sample numbers. Analyses were performed using GraphPad Prism 8.0.1., and $p<0.05$ was considered to be statistically significant.

\section{Results}

\section{RA Patients Showed Disrupted Albumin and Globulin Levels}

The epidemiological characteristics of all RA patients and $\mathrm{HC}$ are given in Supplementary Table 2. Compared to HC, RA patients had decreased albumin levels but with an increase in globulin levels, which led to a significantly 



Figure I Disturbed albumin and globulin levels in RA patients have significantly correlated with age and GFR. $(\mathbf{A}-\mathbf{C})$ Compared to HC $(n=38)$, RA patients $(n=134)$ had decreased albumin levels, while an increase in globulin levels was observed. Significantly lower A/G ratio was present in RA patients. (D) Albumin values negatively correlated with age in RA patients. (E) Albumin values positively correlated with GFR in RA patients. (F) A/G ratio had a significant negative correlation with age. (G) Albumin level also showed a negative correlation with age in $\mathrm{HC}$. $* * \mathrm{p}<0.01$; ****p $<0.000 \mathrm{I}$.

lower A/G ratio (Figure $1 \mathrm{~A}-\mathrm{C}$ ). Consistent with our previous observations, ${ }^{21}$ albumin and globulin levels have inversely correlated with disease activity. Contrary to globulin levels, albumin values negatively correlated with ESR and CRP levels (Table 1). Therefore, A/G ratio had a significant negative correlation with ESR and CRP values (Table 1).

A mild negative correlation between albumin levels and age and a positive correlation with glomerular filtration rate (GFR) were observed in RA patients (Figure 1D and E). However, age and GFR showed a comparatively strong negative correlation (Figure 1F). This indicates the decreasing trend in albumin synthesis with age, which was confirmed in $\mathrm{HC}$ as well (Figure $1 \mathrm{G}$ ).

\section{Albumin and Globulin Levels Correlated with Dyslipidemia in RA}

LDL level did not show any significant differences between HC and RA, but both TC and HDL levels were higher in $\mathrm{HC}$ than RA (Figure $2 \mathrm{~A}-\mathrm{C}$ ). These results are in accordance with an earlier large scale cohort study. ${ }^{22}$ Although in RA patients cholesterol, especially LDL, is a risk for disease flare-up and for complications accompanying AID, lower levels of TC and HDL might be due to 
Table I Correlations Between Albumin, Globulin and A/G Ratio with Levels of ESR, CRP, Serum Lipids and Cytokines

\begin{tabular}{|c|c|c|c|c|c|c|c|c|c|}
\hline \multirow[t]{2}{*}{ Variables } & \multicolumn{3}{|c|}{ Albumin (g/L) } & \multicolumn{3}{|c|}{ Globulin (g/L) } & \multicolumn{3}{|c|}{ A/G } \\
\hline & $r$ & $95 \% \mathrm{Cl}$ & $p$-value & $r$ & $95 \% \mathrm{Cl}$ & $p$-value & $r$ & $95 \% \mathrm{Cl}$ & $p$-value \\
\hline Age (y/o) & 0.22 & {$[-0.35,-0.08]$} & 0.002 & -0.02 & {$[-0.16,0.1 \mathrm{I}]$} & 0.75 & -0.06 & {$[-0.20,0.06]$} & 0.32 \\
\hline ESR $(\mathrm{mm} / \mathrm{h})$ & -0.46 & {$[-0.56,-0.34]$} & $<0.0001$ & 0.53 & {$[0.42,0.62]$} & $<0.0001$ & -0.56 & {$[-0.65,-0.46]$} & $<0.000$ I \\
\hline CRP $(\mathrm{mg} / \mathrm{L})$ & -0.38 & {$[-0.49,-0.25]$} & $<0.0001$ & 0.25 & {$[0.11,0.37]$} & 0.0004 & -0.37 & {$[-0.48,-0.24]$} & $<0.000$ I \\
\hline $\mathrm{TC}(\mathrm{mmol} / \mathrm{L})$ & 0.32 & {$[0.16,0.047]$} & 0.0001 & -0.26 & {$[-0.41,-0.09]$} & 0.0022 & 0.44 & {$[0.29,0.57]$} & $<0.000$ I \\
\hline LDL (mmol/L) & 0.26 & {$[0.09,0.4 I]$} & 0.0025 & -0.14 & {$[-0.30,0.03]$} & 0.1148 & 0.27 & {$[0.10,0.42]$} & 0.0016 \\
\hline TG (mmol/L) & 0.15 & {$[-0.01,0.32]$} & 0.0673 & 0.06 & {$[-0.10,0.23]$} & 0.4634 & 0.03 & {$[-0.13,0.20]$} & 0.6794 \\
\hline $\mathrm{HDL}(\mathrm{mmol} / \mathrm{L})$ & 0.23 & {$[0.06,0.38]$} & 0.0078 & -0.28 & {$[-0.43,-0.12]$} & 0.0008 & 0.40 & {$[0.25,0.53]$} & $<0.000$ I \\
\hline Leptin (mRNA) & 0.12 & {$[-0.05,0.29]$} & 0.1684 & -0.24 & {$[-0.40,-0.07]$} & 0.0053 & 0.17 & {$[0.00,0.34]$} & 0.0475 \\
\hline $\operatorname{Tr} 2$ (mRNA) & 0.09 & {$[-0.08,0.26]$} & 0.2876 & -0.18 & {$[-0.34,-0.01]$} & 0.0385 & 0.28 & {$[0.11,0.43]$} & 0.0014 \\
\hline IL-6 (pg/mL) & -0.34 & {$[-0.52,-0.13]$} & 0.0017 & 0.13 & {$[-0.08,0.34]$} & 0.2268 & -0.31 & {$[-0.50,-0.10]$} & 0.0040 \\
\hline IL-8 (pg/mL) & -0.44 & {$[-0.60,-0.24]$} & $<0.0001$ & 0.26 & {$[0.04,0.45]$} & 0.0167 & -0.44 & {$[-0.60,-0.24]$} & $<0.000$ I \\
\hline TNF-a (pg/mL) & -0.51 & {$[-0.65,-0.33]$} & $<0.0001$ & 0.31 & {$[0.10,0.50]$} & 0.0040 & -0.46 & {$[-0.62,-0.27]$} & $<0.000$ I \\
\hline
\end{tabular}

Notes: The bold $p$-values are less than 0.05 , and indicate significant differences.

Abbreviations: A/G ratio, albumin to globulin ratio; CRP, C-reactive protein; ESR, erythrocyte sedimentation rate; TC, total cholesterol; HDL, high-density lipoprotein; LDL, low-density lipoprotein; TG, triglyceride; IL, interleukin; TNF-a, tumor necrosis factor-a.

poor nutritional status prevailing in this RA population, as indicated by the observed rheumatoid cachexia. ${ }^{23}$ Albumin showed a positive correlation with LDL, TC and HDL levels. Conversely, globulin levels showed a negative correlation with TC and HDL levels (Table 1). Apparently, A/ $\mathrm{G}$ ratio has a more sensitive positive correlation with LDL, TC and HDL levels (Table 1). However, these correlations were not seen in HC (Supplementary Figure 3), which may suggest inflammation led disturbances in $\mathrm{A} / \mathrm{G}$ ratio and dyslipidemia, which had an interdependent relation.

Furthermore, except the mild correlation observed between CRP and TC, levels of other lipids like LDL, TC and HDL rarely showed any correlation with ESR and CRP (Figure 2D and E), hallmarks of an acute inflammation.

\section{Decreased A/G Ratio is a Presumptive Marker for ARDs in RA}

Since $\mathrm{A} / \mathrm{G}$ ratio showed a significant negative correlation with inflammatory activity, while a positive correlation with HDL levels prevailed, it is plausible for decreased $\mathrm{A} / \mathrm{G}$ ratio to become a hallmark for vascular diseases. Similar to general CVD population, RA patients with CVD also had a higher age range compared to patients without CVD (Figure 3A). And according to our above results, age is a factor that affected albumin levels in both RA and HC. So, we excluded patients who were under 50 years, to get age-matched data of patients with or without CVD (Figure 3B). In these two groups of patients, although TC and LDL levels did not have any significant differences (Supplementary Table 3), HDL level was lower in RA patients with CVD (Figure 3C). Because of the higher globulin levels present in these RA patients with CVD than without CVD, lower $\mathrm{A} / \mathrm{G}$ ratio was observed in the RA patients with CVD (Figure 3D-F). Again, acute inflammatory markers like ESR and CRP did not show any significant differences (Figure $3 \mathrm{G}$ and $\mathrm{H}$ ).

Besides 16 patients diagnosed with CVD, there were 8 patients with diabetes, 5 with cancer ( 3 with lung cancer and 2 with colon cancer) and 26 patients with osteoporosis. In total, 44 patients were diagnosed with these ARDs as there were overlapping diseases in some patients (Figure 3I). Patients with $\mathrm{A} / \mathrm{G}<1.2$ had a higher risk of ARDs $(36 / 56$, $64.3 \%)$ than those patients with $\mathrm{A} / \mathrm{G}>1.2(9 / 78,11.5 \%)$ (Figure 3J). The relative risk was 2.48 (95\% CI:1.79 to $3.64, \mathrm{p}<0.0001$ ), with sensitivity and specificity of $\mathrm{A} / \mathrm{G}$ ratio for ARDs in RA of $77.5 \%$ and $80.0 \%$, respectively.

\section{A/G Ratio Had a Positive Correlation with the Expression of Leptin and Trf2}

In this study, expression of leptin but not Trf2 was found to be increased in RA patients (Figure 4A and B). The 


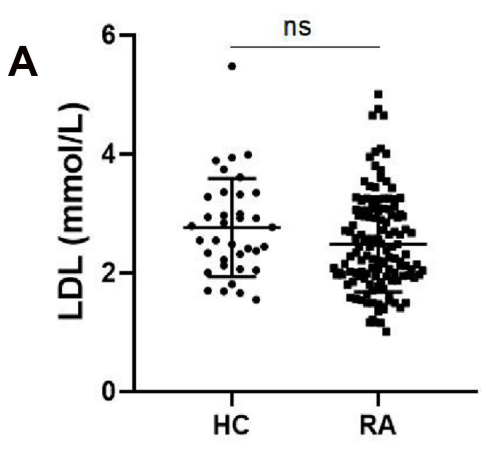

B

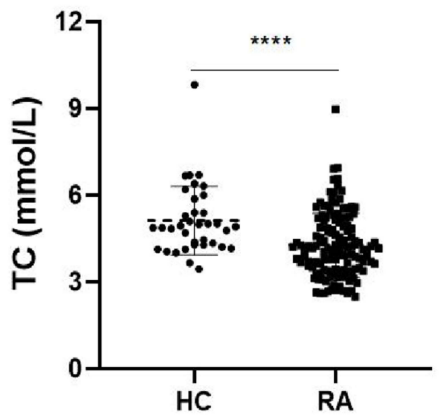

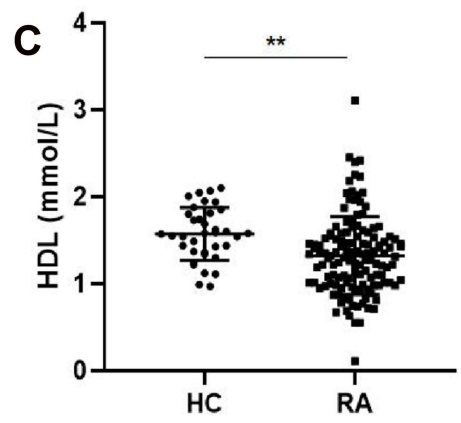
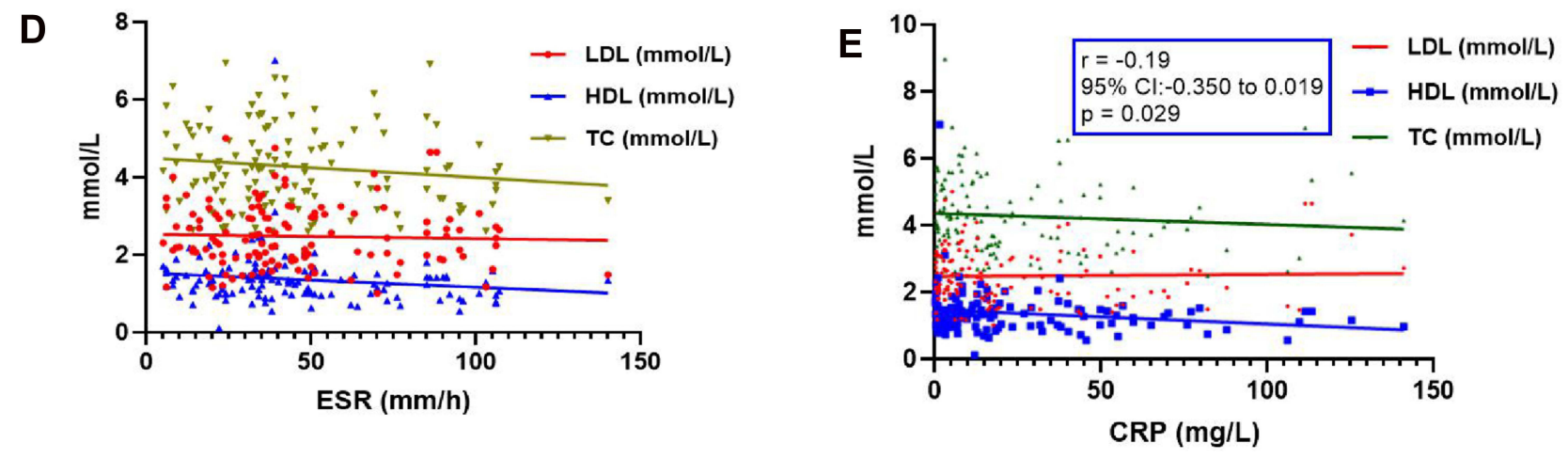

Figure 2 Dyslipidemia in RA. (A-C) LDL level did not differ significantly between HC and RA, but both TC and HDL levels were higher in HC than RA. (D and E) CRP and TC had a negative correlation, whereas LDL, TC and HDL rarely showed any correlation with ESR and CRP. ns, not significant; ** $<<0.0 \mathrm{I}$; ***** $<0.000 \mathrm{I}$.

increased leptin levels showed a moderate positive correlation with both $\mathrm{TC}$ and HDL levels in RA patients (Figure 4C). Because of the negative correlation of leptin to globulin levels, a positive correlation to $A / G$ ratio was observed (Table 1). Although the expression of $\operatorname{Trf} 2$ decreased in RA patients, the variation trends with globulin and $\mathrm{A} / \mathrm{G}$ ratio were similar to leptin mRNA levels (Table 1) and both of them showed a strong positive correlation between themselves (Figure 4D) in RA patients.

\section{Albumin and Globulin Levels Significantly Correlated with Inflamm-Aging Related Cytokines}

As pro-inflammatory cytokines are proposed to be related to the inflamm-aging process, our interest was aroused by the presence of dyslipidemia and increased leptin mRNA levels in RA patients, which led us to assay for the level of adipokines. Therefore, an endocrine multiplex assay focused on adipokines was performed and an increase in the plasma levels of NGF, MCP-1, IL-1, IL-6, IL-8 and TNF-a levels in RA $(n=46)$ compared to HC $(n=38)$ individuals were detected (features of the two groups are given in Supplementary Table 4). While insulin and leptin levels failed to show any significant differences (Figure 5A and B), in RA patients, an increased IL-6 level showed a significant negative correlation with albumin levels and a negative correlation with $\mathrm{A} / \mathrm{G}$ ratio (Figure 5C, Table 1). Both IL-8 and TNF-a levels had a negative correlation with albumin levels while a positive correlation was detected with globulin levels, which led to a negative correlation with $\mathrm{A} / \mathrm{G}$ ratio (Figure 5D and E and Table 1). IL-1, MCP-1 and NGF also increased in RA patients (Figure 5F-H), but with neither any significant correlation with albumin and globulin nor $A / G$ levels in this study.

\section{Discussion}

Pathogenesis of RA, including many other AIDs, involves an abnormal inflammation and metabolism profile, ${ }^{1}$ which may contribute to an accelerated process of aging and an early disease onset. Incidence of diabetes mellitus, osteoporosis, malignancies and cardiovascular diseases among RA patients is significantly higher compared to non-rheumatic controls. ${ }^{3,24}$ One of the novel 



Figure 3 Decreased A/G ratio as a presumptive marker for cardiovascular diseases and other ARDs in RA patients. (A) RA patients with CVD had a higher age range than those without CVD. (B) Age-matched data of patients with or without CVD. (C) HDL is lower in age-matched RA with CVD patients. (D-F) Higher globulin levels in RA patients with CVD manifested lower A/G ratio. ( $\mathbf{G}$ and $\mathbf{H}$ ), ESR and CRP had no significant differences between RA with or without CVD. (I) I6 patients with CVD, 8 with diabetes, 5 with cancer and 26 with OP were used in this study. In total, 44 patients were diagnosed with these ARDs. (J) Patients with A/G <I.2 had a higher risk for ARDs than patients with $\mathrm{A} / \mathrm{G}>1.2$. ns, not significant; *p $<0.05$; **p $<0.01$; ****p $<0.000$ I.

concepts to interpret the underlying mechanism is inflammaging. Inflammaging and metaflammation (the metabolic inflammation accompanying metabolic diseases) largely share similar molecular mechanisms. ${ }^{25}$ However, inflammaging may not be the trigger for clinical manifestations of ARDs, because it is not the main cause for the development of these diseases. ${ }^{26}$ It has been stressed that a chronic, low-grade inflammation could be the main reason for aging and development of ARDs. ${ }^{15,25,27}$ On the other hand, an acute inflammation is rarely considered to be a significant factor. Thus, the inflammatory indexes such as ESR, CRP and also levels of cytokines like TNFa, IL-17 and IL-6 may not demonstrate the inflammation status in mild and chronic disease forms. Hence, new biomarkers like DNA methylation, glycomics, metabolomics and lipidomics that are capable of assessing biological versus chronological age were proposed. ${ }^{25}$ Obviously, these biomarkers are not practically used in current daily clinical work.

Serum albumin levels and $\mathrm{A} / \mathrm{G}$ ratio are being investigated in relation to inflammation and applied as indexes for systemic inflammation in various diseases such as sepsis, SLE and RA. ${ }^{28-31}$ Albumin and A/G levels were reported to be diminished in $\mathrm{RA}$, and also inversely correlated with disease activity, ${ }^{31,32}$ which was confirmed by our current investigation. Although, the glucose and lipid metabolic disturbances leading to insulin resistance are strongly associated with the degree of systemic inflammation, and glycolysis is activated in RA under proinflammatory conditions, ${ }^{33,34}$ our study showed a decrease in LDL level but without any statistical significance, contrary to TC and HDL levels in RA, which were decreased with statistical significance. The $A / G$ ratio has negatively correlated with inflammation while positively 



Figure 4 Relative expression of leptin and Trf2 mRNA levels in RA. (A) The relative expression of leptin mRNA level was increased in RA patients. (B) The relative expression of Trf2 mRNA level was decreased in RA patients. (C) An increased leptin level showed a moderate positive correlation with both TC and HDL levels in RA patients. (D) Leptin and Trf2 expression levels showed a strong positive correlation. ns, not significant; ${ }^{*} \mathrm{p}<0.05$; ${ }^{* *} \mathrm{p}<0.01$.

correlated with lipid levels, which also contributed to the inflammation seems to be controversial. Decreased cholesterol and lipoprotein levels in RA were in accordance with other clinical findings, ${ }^{22,35}$ although high lipid profile contributing to inflammation is also profoundly reported in basic research. ${ }^{36,37}$ This phenomenon can be explained by the pathogenesis of rheumatoid cachexia, ${ }^{23}$ and an increased cholesterol level was regarded as one of the benefits from treatment in RA patients. ${ }^{38}$ Furthermore, serum albumin and $\mathrm{A} / \mathrm{G}$ levels have positively correlated with TC, LDL and HDL levels in this study, while inflammatory indexes like ESR and CRP did not have any such sensitive correlation, except a mild negative correlation between CRP and HDL. Both ESR and CRP had no significant differences between RA patients with and without CVD, while serum globulin levels have significantly increased with a subsequent decrease in $\mathrm{A} / \mathrm{G}$ ratio. These results further confirmed the theory that inflammaging is 

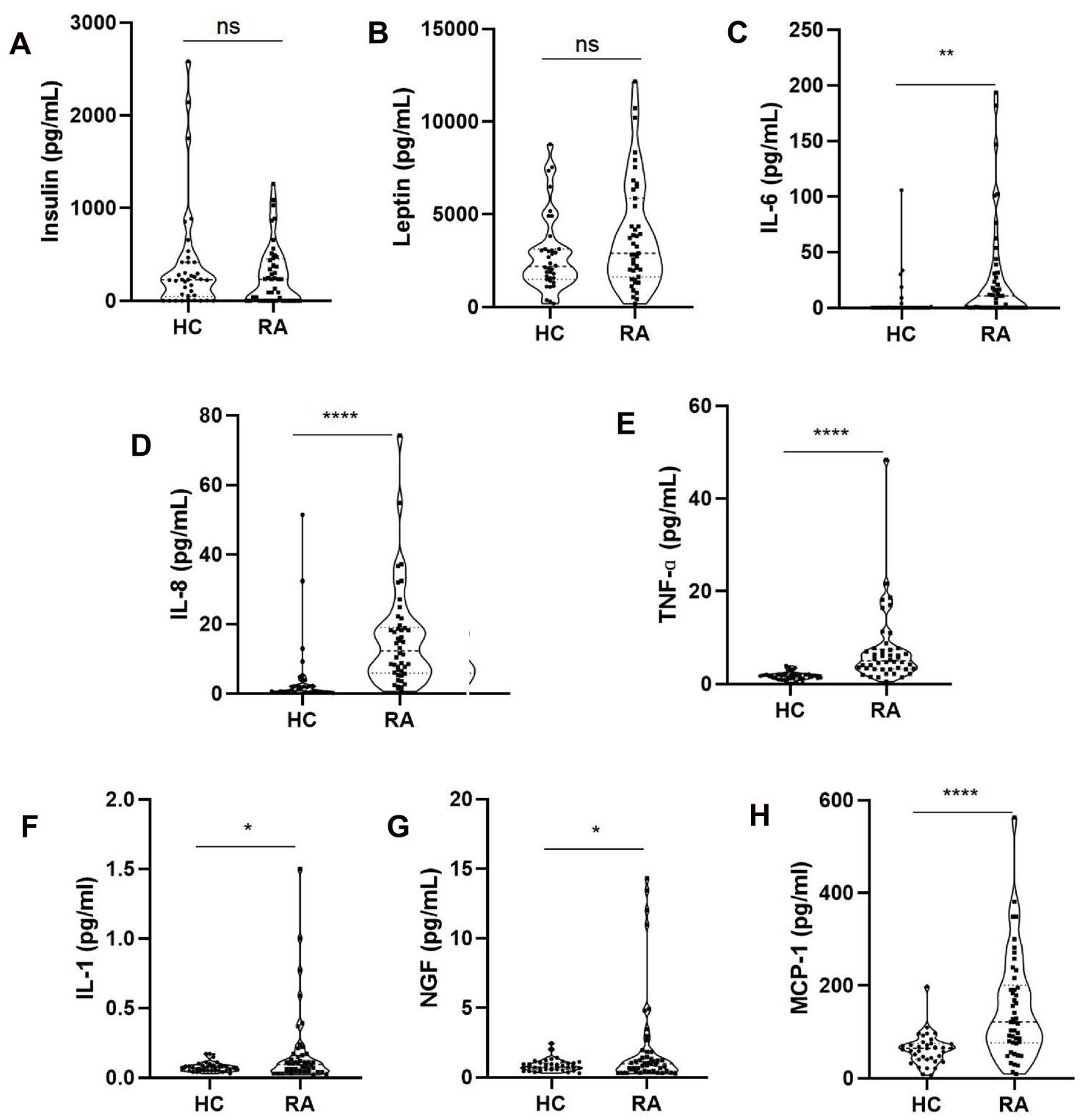

Figure 5 Inflamm-aging related cytokines increased in RA patients. (A and B) No significant differences between HC and RA in the insulin and leptin levels. (C-H) IL-6, IL-8, TNF-a, NGF, IL-I, NGF and MCP-I levels were increased in RA patients. ns, not significant; *p <0.05; **p <0.0I; ****p <0.000I.

indeed the result of chronic but not acute inflammation. This study also demonstrated that a decrease in $\mathrm{A} / \mathrm{G}$ ratio is a risk factor for incidence of ARDs in RA.

As a consequence of its dual role as an anorexigenic and a pro-inflammatory factor, leptin is considered as a link between the neuroendocrine and immune systems. $^{5,39}$ In this study, leptin expression showed a mild positive correlation with TC and HDL levels in RA. It is of interest to note that the down-regulated Trf2 mRNA levels in RA patients showed a strong positive correlation with the up-regulated leptin mRNA expression, and both of them showed a mild positive correlation with the $\mathrm{A} / \mathrm{G}$ ratio. Trf2 plays an important role in telomere maintenance, ${ }^{6}$ which closely relates to biological age, and, importantly, shortening of telomere length was observed in RA patients. Thus, the positive correlations between serum albumin, $A / G$ ratio, $\operatorname{Tr} 2$ and leptin expression levels indicate reliability of $\mathrm{A} / \mathrm{G}$ ratio in representing the aging process and aging-related molecules.

Apart from the elevated levels of well-known cytokines like IL-1, IL-6, IL-8 and TNF-a, both NGF and MCP-1 were also significantly elevated in the plasma of RA patients. NGF and MCP-1 were earlier reported to be up-regulated in the synovial tissue of RA patients. ${ }^{40,41}$ Unlike serum globulin levels, which showed a positive correlation, albumin and $A / G$ ratio have shown a negative correlation with 
IL-6, IL-8 and TNF-a levels, which participate in the inflammaging processes. ${ }^{42}$ These results confirmed our hypothesis of using $\mathrm{A} / \mathrm{G}$ ratio as a marker for evaluating inflammaging. Based on our findings, a diagram showing cause-effect between immunosenescence and aging contributing to RA pathogenesis was shown (Supplementary Figure 4).

However, this study has several limitations. Firstly, a large portion of RA patients enrolled were comparatively old, which might cause bias for higher incidence of ARDs in RA. Secondly, as with glucocorticoid therapy in RA, it is hard to exclude OP perpetuated by inflammaging and prescribed medicines. ${ }^{43}$ Thirdly, A/G ratio might be used for prognosis of various cancers, ${ }^{44-46}$ but this observational study did not address this question in RA. Hence, further longitudinal studies are needed to evaluate the usefulness of $\mathrm{A} /$ $\mathrm{G}$ ratio.

\section{Conclusion}

The ancient Yin-Yang theory is widely applied today to explain opposite but inter-dependent matters and imbalance within our body. ${ }^{4,48}$ Decreased serum albumin values with an increase in globulin levels observed in RA is in accordance with the TCM school of thought that advocates insufficient Yin while excess Yang is the common factor facilitating pathogenesis shared by most disorders. Our research determined a significant correlation between decreased $\mathrm{A} / \mathrm{G}$ ratio and dyslipidemia as well as ARDs in RA patients. By analyzing the correlation between $\mathrm{A} / \mathrm{G}$ ratio and inflammaging-related adipokines and pro-inflammatory cytokines, we recommend albumin as Yin, globulin as Yang and $\mathrm{A} / \mathrm{G}$ ratio as a reliable marker for inflammaging process during clinical management.

\section{Acknowledgments}

We thank the patients who have participated in the study, and we are grateful for the time and effort from all the investigators, doctors, study nurses, and research assistants at the study centers for their invaluable contribution to this study.

\section{Funding}

This study was funded by the National Natural Science Foundation of China (Project No. 8197060898), and the National Key Research and Development Program of China (2019YFC0840603).

\section{Disclosure}

The authors report no conflicts of interest in this work.

\section{References}

1. Chen Y, Qiu F, Yu B, et al. Metformin, an AMPK activator, inhibits activation of FLSs but promotes HAPLN1 secretion. Mol Ther Methods Clin Dev. 2020:1202-1214. doi:10.1016/j.omtm.2020.05.008.

2. Fulop T, Larbi A, Hirokawa K, Cohen AA, Witkowski JM. Immunosenescence is both functional/adaptive and dysfunctional/ maladaptive. Semin Immunopathol. 2020;42(5):521-536. doi:10.1007/s00281-020-00818-9

3. Solomon DH, Love TJ, Canning C, Schneeweiss S. Risk of diabetes among patients with rheumatoid arthritis, psoriatic arthritis and psoriasis. Ann Rheum Dis. 2010;69(12):2114-2117. doi:10.1136/ard.2009.125476

4. Chen Y, Cai JF, Lin CH, Guan JL. Demography of vascular Behcet's disease with different gender and age: an investigation with 166 Chinese patients. Orphanet $J$ Rare Dis. 2019;14(1):88-95. doi:10.1186/s13023-019-1061-1

5. Navarini L, Margiotta D, Vadacca M, Afeltra A. Leptin in autoimmune mechanisms of systemic rheumatic diseases. Cancer Lett. 2018;423:139-146. doi:10.1016/j.canlet.2018.03.011

6. Ruis P, Van Ly D, Borel V, et al. TRF2-independent chromosome end protection during pluripotency. Nature. 2021;589(7840):103-109. doi:10.1038/s41586-020-2960-y

7. Flynn MG, Markofski MM, Carrillo AE. Elevated inflammatory status and increased risk of chronic disease in chronological aging: inflamm-aging or inflamm-inactivity? Aging Dis. 2019;10 (1):147-156. doi:10.14336/AD.2018.0326

8. Soeters PB, Wolfe RR, Shenkin A. Hypoalbuminemia: pathogenesis and clinical significance. JPEN J Parenter Enteral Nutr. 2019;43 (2):181-193. doi:10.1002/jpen.1451

9. Maurer M, Altrichter S, Schmetzer O, Scheffel J, Church MK, Metz M. Immunoglobulin E-Mediated autoimmunity. Front Immunol. 2018;9:689. doi:10.3389/fimmu.2018.00689

10. Li K, Fu W, Bo Y, Zhu Y. Effect of albumin-globulin score and albumin to globulin ratio on survival in patients with heart failure: a retrospective cohort study in China. BMJ Open. 2018;8(7):e22960. doi:10.1136/bmjopen-2018-022960

11. Xiao S, Feng F, Liu N, et al. Preoperative albumin level is superior to Albumin-Globulin ratio as a predicting indicator in gastric cancer patients who underwent curative resection. Cancer Manag Res. 2019;11:9931-9938. doi:10.2147/CMAR.S230741

12. Wu PP, Hsieh YP, Kor CT, Chiu PF. Association between Albumin-Globulin ratio and mortality in patients with chronic kidney disease. J Clin Med. 2019;8(11):1991-2003. doi:10.3390/jcm8111991

13. Maeda S, Takeya Y, Oguro R, et al. Serum albumin/globulin ratio is associated with cognitive function in community-dwelling older people: the Septuagenarians, Octogenarians, Nonagenarians Investigation with Centenarians study. Geriatr Gerontol Int. 2019;19(10):967-971. doi:10.1111/ggi.13751

14. Qin J, Qin Y, Wu Y, et al. Application of albumin/globulin ratio in elderly patients with acute exacerbation of chronic obstructive pulmonary disease. $J$ Thorac Dis. 2018;10(8):4923-4930. doi:10.21037/ jtd.2018.07.47

15. Franceschi C, Campisi J. Chronic inflammation (inflammaging) and its potential contribution to age-associated diseases. J Gerontol A Biol Sci Med Sci. 2014;69(Suppl 1):S4-S9. doi:10.1093/gerona/glu057

16. Ferrucci L, Fabbri E. Inflammageing: chronic inflammation in ageing, cardiovascular disease, and frailty. Nat Rev Cardiol. 2018;15 (9):505-522. doi:10.1038/s41569-018-0064-2

17. Simonis F. Illness, texts, and "Schools" in Danxi medicine: a new look at Chinese Medical History from 1320 to 1800. In: Elman BA, editor. Antiquarianism, Language, and Medical Philology. Boston: Brill; 2015:52-80.

18. Lulkiewicz M, Bajsert J, Kopczynski P, Barczak W, Rubis B. Telomere length: how the length makes a difference. Mol Biol Rep. 2020;47(9):7181-7188. doi:10.1007/s11033-020-05551-y 
19. Shin S, Park EH, Kang EH, Lee YJ, Song YW, Ha YJ. Sex differences in clinical characteristics and their influence on clinical outcomes in an observational cohort of patients with rheumatoid arthritis. Joint Bone Spine. 2020. doi:10.1016/j.jbspin.2020.105124

20. Aletaha D, Neogi T, Silman AJ, et al. Rheumatoid arthritis classification criteria: an American College of Rheumatology/European League against Rheumatism collaborative initiative. Arthritis Rheum. 2010;62(9):2569-2581. doi:10.1002/art.27584

21. Yong C, Yanan X, Yanjuan C, et al. Correlation of albumin globulin ratio with DAS28 and ESR levels in rheumatoid arthritis patients. J Jinan Univ. 2019;40(5):373-382. doi:10.11778/j.jdxb.2019.05.001

22. Liao KP, Cai T, Gainer VS, et al. Lipid and lipoprotein levels and trend in rheumatoid arthritis compared to the general population. Arthritis Care Res. 2013;65(12):2046-2050. doi:10.1002/acr.22091

23. Santo R, Fernandes KZ, Lora PS, Filippin LI, Xavier RM. Prevalence of rheumatoid cachexia in rheumatoid arthritis: a systematic review and meta-analysis. J Cachexia Sarcopenia Muscle. 2018;9 (5):816-825. doi:10.1002/jcsm.12320

24. Crowson CS, Rollefstad S, Ikdahl E, et al. Impact of risk factors associated with cardiovascular outcomes in patients with rheumatoid arthritis. Ann Rheum Dis. 2018;77(1):48-54. doi:10.1136/annrheumdis-2017-211735

25. Franceschi C, Garagnani P, Parini P, Giuliani C, Santoro A. Inflammaging: a new immune-metabolic viewpoint for age-related diseases. Nat Rev Endocrinol. 2018;14(10):576-590. doi:10.1038/ s41574-018-0059-4

26. Fulop T, Larbi A, Witkowski JM. Human inflammaging. Gerontology. 2019;65(5):495-504. doi:10.1159/000497375

27. Howcroft TK, Campisi J, Louis GB, et al. The role of inflammation in age-related disease. Aging (Albany NY). 2013;5(1):84-93. doi:10.18632/aging.100531

28. Artigas A, Wernerman J, Arroyo V, Vincent JL, Levy M. Role of albumin in diseases associated with severe systemic inflammation: pathophysiologic and clinical evidence in sepsis and in decompensated cirrhosis. $J$ Crit Care. 2016;33:62-70. doi:10.1016/j.jcrc.2015.12.019

29. Idborg H, Eketjall S, Pettersson S, et al. TNF-alpha and plasma albumin as biomarkers of disease activity in systemic lupus erythematosus. Lupus $\mathrm{Sci}$ Med. 2018;5(1):e260. doi:10.1136/lupus-2018-000260

30. Aringer M. Inflammatory markers in systemic lupus erythematosus. J Autoimmun. 2019. doi:10.1016/j.jaut.2019.102374

31. Chen S, Ying H, Du J, et al. The association between albumin-dNLR score and disease activity in patients with rheumatoid arthritis. J Clin Lab Anal. 2019;33(3):e22695. doi:10.1002/jcla.22695

32. He Y, Tang J, Wu B, Yang B, Ou Q, Lin J. Correlation between albumin to fibrinogen ratio, C-reactive protein to albumin ratio and Th17 cells in patients with rheumatoid arthritis. Clin Chim Acta. 2020;500:149-154. doi:10.1016/j.cca.2019.10.009

33. Arias DLRI, Escudero-Contreras A, Rodriguez-Cuenca S, et al. Defective glucose and lipid metabolism in rheumatoid arthritis is determined by chronic inflammation in metabolic tissues. $J$ Intern Med. 2018;284(1):61-77. doi:10.1111/joim.12743
34. Garcia-Carbonell R, Divakaruni AS, Lodi A, et al. Critical role of glucose metabolism in rheumatoid arthritis fibroblast-like synoviocytes. Arthritis Rheumatol. 2016;68(7):1614-1626. doi:10.1002/art.39608

35. Bag-Ozbek A, Giles JT. Inflammation, adiposity, and atherogenic dyslipidemia in rheumatoid arthritis: is there a paradoxical relationship? Curr Allergy Asthma Rep. 2015;15(2):497-510. doi:10.1007/s11882-014-0497-6

36. Poznyak A, Grechko AV, Poggio P, Myasoedova VA, Alfieri V, Orekhov AN. The diabetes Mellitus-Atherosclerosis connection: the role of lipid and glucose metabolism and chronic inflammation. Int J Mol Sci. 2020;21(5):1835-1848. doi:10.3390/ijms21051835

37. Zhang $\mathrm{C}$, Wang $\mathrm{K}$, Yang $\mathrm{L}$, et al. Lipid metabolism in inflammation-related diseases. Analyst. 2018;143(19):4526-4536. doi:10.1039/c8an01046c

38. Lukas C, Redondin M, Pane I, et al. Cardiovascular events and change in cholesterol levels in patients with rheumatoid arthritis treated with tocilizumab: data from the REGATE registry. Clin Exp Rheumatol. 2021;39(3):501-507.

39. Abella V, Scotece M, Conde J, et al. Leptin in the interplay of inflammation, metabolism and immune system disorders. Nat Rev Rheumatol. 2017;13(2):100-109. doi:10.1038/nrrheum.2016.209

40. Barthel C, Yeremenko N, Jacobs R, et al. Nerve growth factor and receptor expression in rheumatoid arthritis and spondyloarthritis. Arthritis Res Ther. 2009;11(3):R82. doi:10.1186/ar2716

41. An Q, Yan W, Zhao Y, Yu K. Enhanced neutrophil autophagy and increased concentrations of IL-6, IL-8, IL-10 and MCP-1 in rheumatoid arthritis. Int Immunopharmacol. 2018;65:119-128. doi:10.1016/ j.intimp.2018.09.011

42. Rea IM, Gibson DS, Mcgilligan V, Mcnerlan SE, Alexander HD, Ross OA. Age and age-related diseases: role of inflammation triggers and cytokines. Front Immunol. 2018. doi:10.3389/fimmu.2018.00586

43. Adami G, Saag KG. Osteoporosis pathophysiology, epidemiology, and screening in rheumatoid arthritis. Curr Rheumatol Rep. 2019;21 (7):34-43. doi:10.1007/s11926-019-0836-7

44. Guo X, Shao J, Zhai B, et al. Relationship and prognostic significance between preoperative serum albumin to globulin ratio and $\mathrm{CT}$ features of non-small cell lung cancer. Eur J Radiol. 2020. doi:10.1016/j.ejrad.2020.109039

45. Bozkaya Y, Erdem GU, Demirci NS, et al. Prognostic importance of the albumin to globulin ratio in metastatic gastric cancer patients. Curr Med Res Opin. 2019;35(2):275-282. doi:10.1080/03007995.2018.1479683

46. Yoshino Y, Taguchi A, Shimizuguchi T, et al. A low albumin to globulin ratio with a high serum globulin level is a prognostic marker for poor survival in cervical cancer patients treated with radiation based therapy. Int J Gynecol Cancer. 2019;29(1):17-22. doi:10.1136/ ijgc-2018-000025

47. Liu RC. Sensory systems: the yin and yang of cortical oxytocin. Nature. 2015;520(7548):444-445. doi:10.1038/nature14386

48. Gonzalez A, Hall MN, Lin SC, Hardie DG. AMPK and TOR: the yin and yang of cellular nutrient sensing and growth control. Cell Metab. 2020;31(3):472-492. doi:10.1016/j.cmet.2020.01.015
Journal of Inflammation Research

\section{Publish your work in this journal}

The Journal of Inflammation Research is an international, peerreviewed open-access journal that welcomes laboratory and clinical findings on the molecular basis, cell biology and pharmacology of inflammation including original research, reviews, symposium reports, hypothesis formation and commentaries on: acute/chronic inflammation; mediators of inflammation; cellular processes; molecular mechanisms; pharmacology and novel anti-inflammatory drugs; clinical conditions involving inflammation. The manuscript management system is completely online and includes a very quick and fair peerreview system. Visit http://www.dovepress.com/testimonials.php to read real quotes from published authors. 